\title{
Erratum to: Momentum meets value investing in a small European market
}

\author{
The Publisher ${ }^{1}$
}

Published online: 20 May 2017

(C) ISEG 2017

\section{Erratum to: Port Econ J \\ DOI 10.1007/s10258-017-0132-2}

The correct article title is presented in this paper and the original article was corrected. The publisher apologizes for this error for which the authors were not responsible. 